\title{
An Efficient and Flexible Window Function for Memristor Model and its Analog Circuit Application
}

\author{
Chandra Prakash Singh, Raghvendra and Saurabh Kumar Pandey
}

\begin{abstract}
The memristor is a nanostructure resistive tuning two terminal novel electronics device that has been widely explored in the area of neuromorphic computing systems, memories, digital circuits, analog circuits and many more new applications. In this article an efficient and flexible window function is presented for linear drift memristor model. Propose window function provides a unique feature (controllable window function discontinuity) to linear drift memristor model by which DPHL (Distorted Pinched Hysteresis Loop) problem is resolved and also improved the programming resistance state of the memristor. Five control parameters are introduced in the presented window function, in order to fix the pre-existing problem (like boundary effect, boundary lock and inflexibility) and make it more flexible. The programmable analog gain amplifier circuit is ultimately executed to instantiate the utilization of evolved memristor model.
\end{abstract}

Index Terms - Window function discontinuity, Control parameter, Boundary effect, Boundary lock, Linear Drift Memristor Model.

\section{INTRODUCTION}

In recent decades, memristor becomes emerging nano-scale electronics device in the area of nanotechnology due to their unique feature of resistive tuning, non-volatility, simple structure, high speed, low cost and COMS-compatibility. Firstly, Chua postulated the concept of memristor by describing the relationship between charge and flux of the device in 1971 and discover the fourth missing fundamental Passive circuit component [1]. First time, in 2008 a team of HP labs fabricated a nano-scale metal-insulator-meta (MIM) structured device using titanium dioxide $\left(\mathrm{TiO}_{2}\right)$ sandwiched between two platinum $(\mathrm{Pt})$ metal electrodes and discovered the resistive switching phenomena [2]. Since then, industries and academia have attracted much more attention to explore the physics behind the resistive switching memristive device.

\footnotetext{
Chandra Prakash Singh, Raghvendra and Saurabh Kumar Pandey are with the Indian Institute of Technology, Patna, India ( e-mail: cps1391993@gmail.com, raghvendra 1821ee06@iitp.ac.in and
} Saurabh@iitp.ac.in )
Therefore, various mathematical concepts have been proposed to describe the resistive switching phenomena in nano-scale memristive device, like Non-linear model [3], Linear Drift model [2], exponential model [4], Simmons tunneling model [5], Threshold adaptive model (TEAM) [6], Variable threshold adaptive model (VTEAM) [7], Conductive filament based model [8] and some SPICE macro models [9][10]. Although, several models for resistive switching device have been proposed in the literature, but any exact model has not been appeared so far. Accordingly, development of memristor model is still an emerging research domain for the research society.

At the first time, a research group from HP lab's proposed a mathematical concept for describing the resistive switching phenomena in memristive device, which is known as linear drift model. In this model, a window function has been included to introduce non-linearity and physical limiting conditions in the device as well as tracking the actual resistive switching activity of the memristive device.

As of now, apart from mathematical analysis of linear drift memristor model, it have also been investigated in numerous applications, for example, as a memory cell in high density nonvolatile random access memory [11], as an artificial synapses in the neuromorphic systems [12], applicable in spiking neural network [13], in multilayer neural network [14], as a programmable resistive element in the analog circuit in which its resistance value can tuned by applied electrical pulses [15]-[17]. In linear drift memristor model, window function is an essential part for capturing the resistive switching dynamics of memristive device. Hence, to develop an appropriate and efficient window function is become crucial aspect for the researchers.

In last few years, various window functions have been analyzed in the literature for linear drift memristor model. Most of the problems associated with reported window functions are boundary effect problem, boundary lock problem, DPHL problem and inflexibility of control parameter problem. In the initial time, different kind of window functions was presented for meristor model by Strukov et al. [2], Joglekar et al. [18], Biolek et al. [19] and Prodromakis et al. [20]. Even though, boundary effect problem and nonlinearity effect were taken care by Strukov, Joglekar and Prodromakis but failed to resolve boundary lock problem. Accordingly, Biolek was presented another window function to fix the boundary lock problem by including device current parameter in the window function, even so this was inflexible. 
In recent past, for the advancement of linear drift memristor model, numerous window functions have been proposed by research community. For instance, Chen et al. [21] proposed a controllable window function to provide better non-linearity, Maldenov et al. [22] proposed a window function with activation threshold, Chowdhury et al. [23] presented a Prodromakis modified trigonometric window function to improve the flexibility of the memristor model, but these window functions have not considered device current parameter, hence these are failed to resolve boundary lock problem. Further, Zha et al. [15,17] and Shi et al.[24] proposed some window function by combining the feature of Biolek and Prodromakis window function, which is able to resolve most of the problem related to window functions, although DPHL problem not have been fixed.

In recent time, some general window function has been proposed by Li et al. [13] and Wen et al. [14] with terribly restricted and more number of control parameter and it has been also analyzed in spiking neural network and multilayer neural networks. After rigorous study of all the reported window function, we can conclude that, window function discontinuity is responsible for resolving boundary lock problem and this is also leads to DPHL problem. Till now, adverse effect of window function discontinuity at the boundary not has been discussed in the literature.

After considering the importance of reported window function and the shortcoming with it, we have proposed an efficient and flexible window function for linear drift memristor model with five control parameters, which is able to fixed boundary lock, boundary effect and inflexibility problem. Additionally, our proposed window function have unique feature to control functional discontinuity by which DPHL problem has been resolve and also improves the memristance resolution, as per author knowledge this has not been examined so far in the literature. Finally, evolved window function has been implemented in programmable analog circuit application and analyzed its performance.

This article is organized as follow. In section 2, basic physics of linear drift memristor model is described along with importance of several reported window functions with their excellence and drawback. In section 3, evaluation of newly proposed window function is done and shown its control parameters range as well as effects over device characteristics. In section 4, evolved memristor model is implemented in gain amplifier analog circuit and examined its performance with respect to window function control parameter. Finally, in section 5 we are concluded our proposed research work.

\section{PHYSICS OF LINEAR DRIFT MODEL WITH WINDOW FUNCTION}

In 2008, a research group from HP Labs manufactured a nanoscale memritive device as well as proposed a mathematical model for describing resistive switching dynamics of the memristive device. In this model, assumes that insulating layer is separated in two regions, one is undoped region and other is oxygen vacancy doped region (more conductive than undoped region) as well as total resistance of the device is calculated as summation of resistance of these two regions. In Fig.1, schematic of linear drift memristor model is given, where total device length is ' $\mathrm{D}$ ', width of the oxygen vacancy doped region is ' $\mathrm{w}(\mathrm{t})$ ', $\mathrm{R}_{1}$ is the resistance of the oxygen vacancy doped region, $R_{2}$ is the resistance of the undoped region and $R_{\text {eq }}$ is the total instantaneous resistance of the device. Moreover, $R_{\mathrm{ON}}$ and $\mathrm{R}_{\mathrm{OFF}}$ is the limiting resistance of the device for fully doped $[\mathrm{w}(\mathrm{t})=\mathrm{D}]$ and fully undoped $[\mathrm{w}(\mathrm{t})=0]$ case and ' $\mathrm{x}=\mathrm{w}(\mathrm{t}) / \mathrm{D}$ ' is the normalized width of the device that is called as internal state parameter and it is restricted between ' 0 ' and ' 1 ' due to physical boundary limitation of the device. The total instantaneous resistance of the nano-scale memristive device can be calculated from expression-1, as mention in [2].

$$
\mathrm{R}_{\mathrm{eq}}=\left\{\mathrm{R}_{\mathrm{ON}} *(\mathrm{x})+\mathrm{R}_{\mathrm{OFF}} *(1-\mathrm{x})\right\}
$$

In this model, when we are exerting electrical stimuli across the device, then drifting of interface between doped and

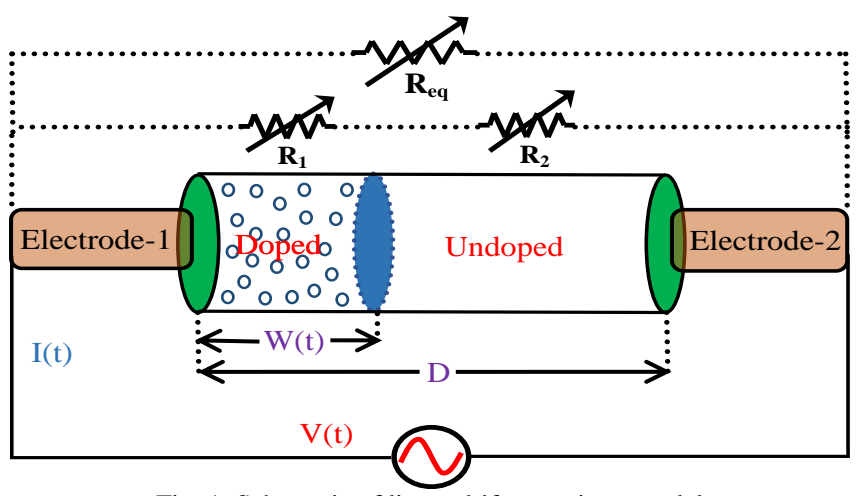

Fig. 1. Schematic of linear drift memristor model.

undoped region occurs. Moreover, the drifting of the interface is formulated as expression-2 by considering basic physics of drift velocity. From expression-2 we can see that, internal state parameter ' $x$ ' is the linear function of charge flow through the device, that is why this model is known as linear drift model.

$\frac{\mathrm{dx}}{\mathrm{dt}}=\mu_{\mathrm{v}} * \frac{\mathrm{R}_{\mathrm{ON}}}{D^{2}} * \mathrm{I}(\mathrm{t})$

Where ' $\mu_{\mathrm{v}}$ ' is the average mobility of oxygen vacancy (dopant) in the proposed memristive device. In this linear drift model, if applying high voltage or low frequency then its doping region goes beyond its physical limit (i.e. boundary effect problem), which is practically impossible. Furthermore, even after exerted small voltage across nano-scale devices it experiences high electric fields, which are leads to nonlinearity in the device characteristics [2]. Accordingly, researchers incorporated a window function in this linear drift model to overcome boundary effect problem and introducing non-linearity in this model. After that, interface dynamic expression is modified as expression-3.

$\frac{\mathrm{dx}}{\mathrm{dt}}=\mu_{\mathrm{v}} * \frac{\mathrm{R}_{\mathrm{ON}}}{D^{2}} * \mathrm{f}(\mathrm{x}) * \mathrm{I}(\mathrm{t})$

To understand the advantages of proposed window function, firstly, we have discussed several reported window function with their properties and drawbacks. First parabolic window function was proposed by Strukov et al. [2], that is given in expression-4. 
$f(x)=x-x^{2}$

This window function is capable to resolves the boundary effect problem, because at the boundary of the device (i.e. $x=1$ or $\mathrm{x}=0$ ) interface drifting become zero with $\mathrm{f}(\mathrm{x})=0$. However, it provides fixed only non-linearity to the device. So, Joglekar et al. [18] proposed another window function with one control parameter to tune the non-linearity of the device, which is shown in expression-5.

$\mathrm{f}(\mathrm{x})=1-(2 \mathrm{x}-1)^{2 \mathrm{p}}$

Where, 'p' should be positive integer only. Even then, it suffers from boundary stick issue, means that, if ones interface hits any boundary of the device ( $\mathrm{x}=0$ or 1 ), it is not capable to tune the resistance of the memristive device from its terminal resistance $\left(\mathrm{R}_{\mathrm{ON}}\right.$ or $\left.\mathrm{R}_{\mathrm{OFF}}\right)$. Thereafter, Biolek et al. [19] proposed a new window function to resolve the boundary stick issue by including device current parameter in window function. This window function is represented by expression6 , which have one control parameter.

$f(x)=1-[x-\operatorname{stp}(-i)]^{2 p}$

Where, ' $\mathrm{p}$ ' is positive integer only and $\operatorname{stp}($.$) is a step$ function which has discontinuity at one point. Additionally, it has restricted flexibility in the window function due to utilizing only one control parameter. By considering above limitations, Prodromakis et al. [20] recommended a parabolic window function with two control parameter which has given in expression-7.

$f(x)=j *\left[1-\left\{(x-0.5)^{2}+0.75\right\}\right]^{p}$

Where, 'p' and ' $j$ ' belongs to positive real number, which provides better flexibility to the window function. But, there is not any device current parameter involved in the window, so it can't handle with boundary stick issue. After taking these constraints into account, an appropriate window function has been provided by Zha et al. [15] with two control parameter including device current in it, which has formulated as expression-8.

$$
f(x)=j *\left[1-\left\{0.25 *(x-\operatorname{stp}(-i))^{2}+0.75\right\}\right]^{p}
$$

Where, ' $p$ ' and ' $\mathrm{j}$ ' are positive real number. This is able to resolve boundary stick issue and also provide good flexibility. Although, this window function model have fixed discontinuity at its boundary, which results in DPHL problem for low frequency or high amplitude exerted external stimuli. To improve the flexibility in Zha et al.[15] window function, Shi et al.[24] proposed another window function model with one additional control parameter $\left(\mathrm{a}_{\mathrm{s}}\right)$, that is responsible for controlling the maximum value of window $\left[f(x)_{\max }\right]$ only up to one, as mentioned in expression-9.

$f(x)=j *\left[1-\left\{a_{s}{ }^{2} *(x-\operatorname{stp}(-i))^{2}+\left(1-a_{s}{ }^{2}\right)\right\}\right]^{p}$

Where ' $p$ ' and ' $\mathrm{j}$ ' are positive real number and $\mathrm{a}_{\mathrm{s}}$ is restricted between ' 0 ' and ' 1 '. Further, Zha et al. [17] again reported another window function with three control parameter, where some of existing window function is special case of this window function, which has given in expression-10. $f(x)=\left[1-\left\{a *(x-\operatorname{stp}(-i))^{2 q}+(1-a)\right\}\right]^{p}$

Where, 'p' and ' $j$ ' are positive real number but ' $q$ ' should be positive integer only and ' $a$ ' is bounded between ' 0 ' and ' 1 '. This window function has resolved most of the problem associated with previous window functions, even than functional discontinuity present here, that is creates DPHL problem(after applying bit high voltage or low frequency input, state variable ' $\mathrm{x}$ ' reaches its limit very quickly which results in distortion in current-voltage response of the device). In addition, this window model has only symmetric nonlinearity at the both end of the device, which is practically not possible always. For address DPHL problem, Anusudha et al. [16] defined a cubic parabolic window function in expression11.

$\mathrm{f}(\mathrm{x})=\mathrm{j} *\left[1-2\left(\mathrm{x}^{3}-\mathrm{x}+1\right)^{\mathrm{p}}\right]$

Where $\mathrm{j} \in \mathrm{R}^{+}$, and ' $\mathrm{p}$ ' is positive integer only. Although, this window function has failed to resolve boundary effect problem, because at the boundary of the device interface drifting is not zero (i.e. at $\mathrm{x}=0$ or $1 ; \mathrm{f}(\mathrm{x}) \neq 0$ ) and it has not discussed the boundary stick issue.

\section{NEWLY PROPOSED WINDOW FUNCTION}

After rigorous analysis of several reported window functions, in this section, we are come up with an efficient and flexible window function model and also discussed about its superiorities over existing window functions. This window function has five control parameters with considering device current, which helps to resolve boundary effect, boundary lock and inflexibility. Proposed window function have a unique property of controlling window function discontinuity, by which it is able to resolves DPHL problem without removing device current parameter from window function and also

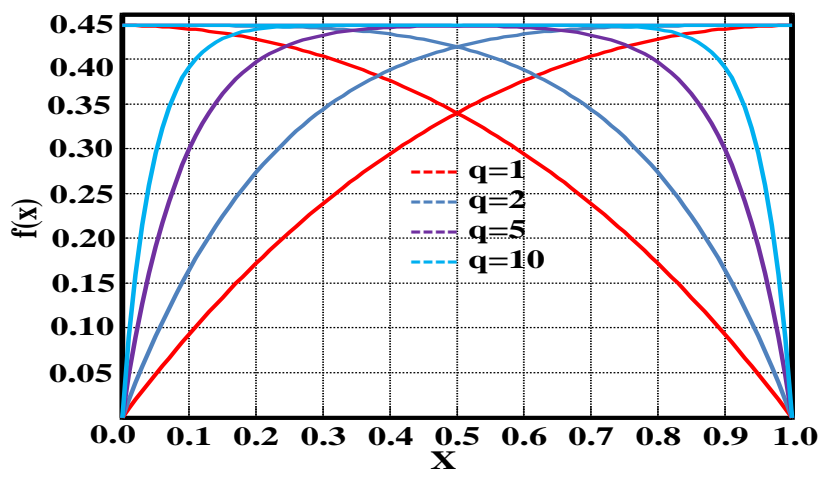

Fig. 2. Effect of ' $q$ ' on proposed window (12) with $\mathrm{a}=0.25, \mathrm{p}=2$ and $\mathrm{j}=1$.

improves the programming resistance state of memristor. It is fulfilled all the necessary criteria of window function which has mention in [17]. Our proposed window function is formulated as expression-12.

$$
\begin{gathered}
f(x)=j *\left[1-\left\{\left(b * x^{2}+a\right) *\{x-\operatorname{stp}(-i)\}^{2 q}\right.\right. \\
\left.\left.+\left(1-a-b * x^{2}\right)\right\}\right]^{p}
\end{gathered}
$$

Where ' $p$ ' and ' $\mathrm{j}$ ' are any positive real number but ' $\mathrm{q}$ ' should be only positive integer. Other two control parameter is 
restricted as $a \in(0,1]$ and $b \in[-a, 0]$ to fulfill the necessity criteria of window function. Proposed window function is able to overcome DPHL problem by controlling the window function discontinuity at their boundary and also improving

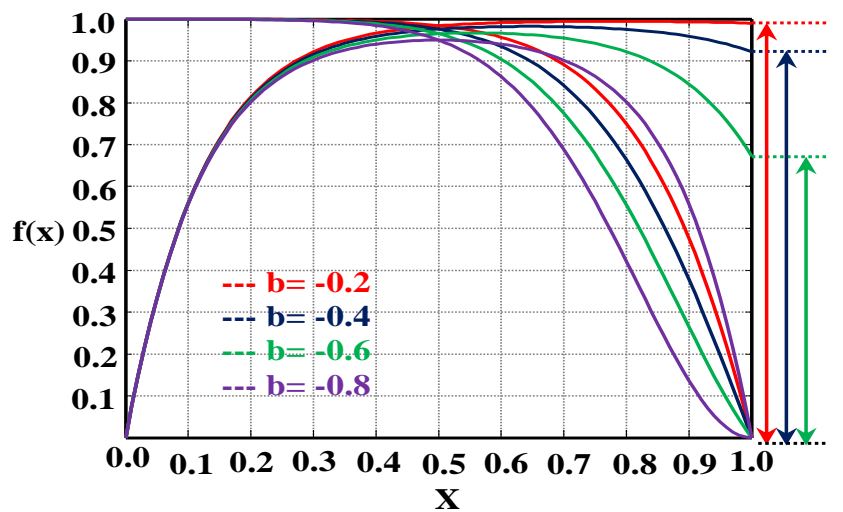

Fig. 3. Effect of 'b' on function discontinuity by proposed window (12) with $\mathrm{a}=0.8, \mathrm{p}=5, \mathrm{q}=1$ and $\mathrm{j}=1$.

programming resistance resolution of memristor, which can enhance the performance of programmable analog circuit in practical implementation.

From Table-1, we can conclude that, some existing window function models are subset of our proposed window function model. Consequently, by taking appropriate value of control parameters we can reproduce some existing window function

TABLE - 1

\section{SUBSET OF PROPOSED WINDOW FUNCTION}

\begin{tabular}{|c|c|}
\hline $\begin{array}{c}\text { Control } \\
\text { Parameter } \\
\text { Value }\end{array}$ & Inherited Window Function \\
\hline $\begin{array}{c}\mathrm{j}=1, \mathrm{a}=1, \mathrm{q}=1, \\
\mathrm{~b}=0 .\end{array}$ & $\mathrm{f}(\mathrm{x})=1-[\mathrm{x}-\operatorname{stp}(-\mathrm{i})]^{2 \mathrm{p}}$ \\
\hline $\begin{array}{c}\mathrm{a}=0.25, \mathrm{q}=1, \\
\mathrm{~b}=0 .\end{array}$ & $\mathrm{f}(\mathrm{x})=\mathrm{j} *\left[1-\left\{0.25 *(\mathrm{x}-\operatorname{stp}(-\mathrm{i}))^{2}+0.75\right\}\right]^{\mathrm{p}}$ \\
\hline $\begin{array}{c}\mathrm{a}=\mathrm{a}_{\mathrm{s}}{ }^{2}, \mathrm{q}=1, \\
\mathrm{~b}=0 .\end{array}$ & $\mathrm{f}(\mathrm{x})=\mathrm{j} *\left[1-\left\{\mathrm{a}_{\mathrm{s}}{ }^{2} *(\mathrm{x}-\operatorname{stp}(-\mathrm{i}))^{2}+\left(1-\mathrm{a}_{\mathrm{s}}{ }^{2}\right)\right\}\right]^{\mathrm{p}}$ \\
\hline $\mathrm{b}=0$. & $\mathrm{f}(\mathrm{x})=\mathrm{j} *\left[1-\left\{\mathrm{a} *(\mathrm{x}-\operatorname{stp}(-\mathrm{i}))^{2 \mathrm{q}}+(1-\mathrm{a})\right\}\right]^{\mathrm{p}}$ \\
\hline
\end{tabular}

$[15,17,19,24]$, hence, properties of these reported window functions are inbuilt in our proposed window function model. The impact of control parameter ' $a$ ', ' $j$ ' and 'p' of memristive model characteristics have been already discussed in $[15,17]$.Thereafter, from Fig. 2 we can observe that, the control parameter ' $q$ ' only modulates non-linearity of the device without affecting maximum value of window function with considering others window control parameters are fixed. When we increase the value of ' $q$ ' then non-linearity of the window is confined to their boundary. Further, we can evident from Fig. 3, the control parameter ' $b$ ' is able to control the functional discontinuity at the boundary of the window function. In next section, we will see by adjusting window function discontinuity at the boundary, DPHL issue has been resolved.

\section{EVALUATION OF PROPOSED WINDOW FUNCTION MODEL}

To evaluate the proposed newly window function we have studied boundary effect issue, boundary stick issue, non- linearity and DPHL issue in the device by taken parameter value as $R_{\mathrm{ON}}=100 \Omega, \mathrm{R}_{\mathrm{OFF}}=16 \mathrm{~K} \Omega, \mathrm{R}_{\text {initial }}=0.5 \mathrm{~K} \Omega, \mathrm{D}=$ $10 \mathrm{~nm}$, and $\mu_{\mathrm{v}}=10^{-14} \mathrm{~m}^{2} \mathrm{~s}^{-1} \mathrm{~V}^{-1}, \mathrm{a}=0.8, \mathrm{p}=5, \mathrm{q}=1, \mathrm{~b}=-0.6$, to simulate in SPICE. Where $R_{\text {initial }}$ is represents the initial resistance of the memristive device.

\subsection{Study of Boundary effect Issue}

We can observed that in Fig. 4, after applying sufficient high

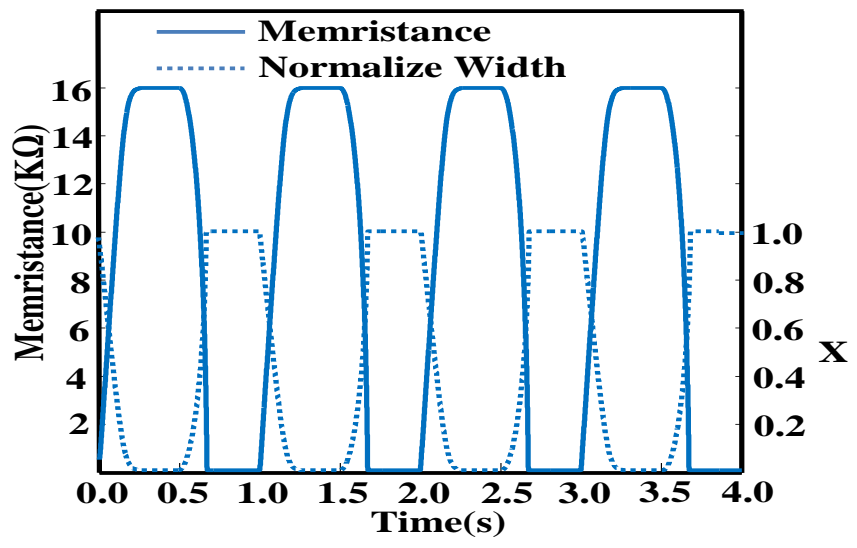

Fig. 4. Response of the normalized width and memristance with high amplitude input voltage

amplitude voltage [like as $\left.\mathrm{Vm}=10 \sin \left(2 \pi \mathrm{f}^{*} \mathrm{t}\right)\right]$ to the memristive device, it operates between its terminal resistance range $\left(\mathrm{R}_{\mathrm{ON}}\right.$ and $\left.\mathrm{R}_{\mathrm{OFF}}\right)$ and never goes beyond their own physical limits (means ' $x$ ' is restricted between 0 to 1 ). Hence, now we can conclude that our proposed window function is free from boundary effect problem.

\subsection{Study of Boundary Stick Issue}

After applying positive and negative sinusoidal voltage to the

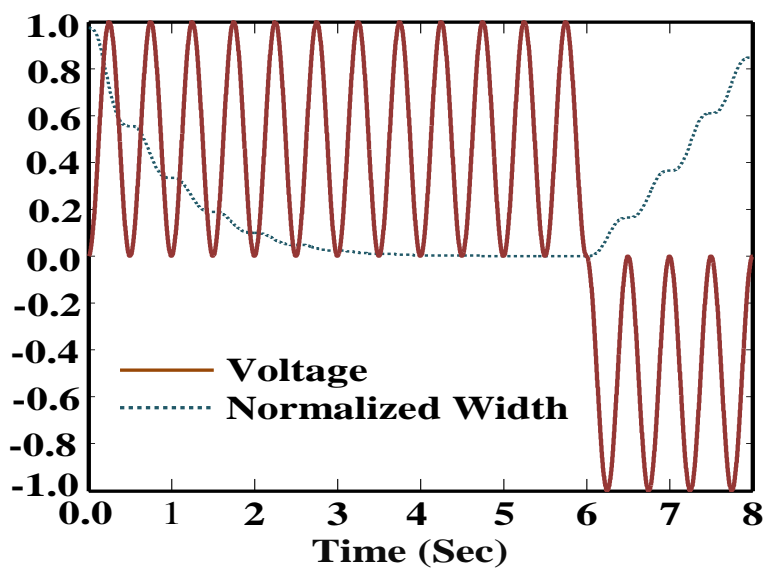

Fig. 5. Response of the normalized width with the input voltage.

memristive device, the response of the normalized width has reported in the Fig. 5. So from Fig. 5, we can observe that, when polarity of the applied voltage changes then its normalized width (state value) modulated from its boundary. Therefore, we can say that our proposed window model is successfully resolved boundary stick issue.

\subsection{Study of DPHL Issue}

When applied electrical stimuli have low frequency or voltage amplitude is high then interface reaches the boundary of the 
TABLE - 2

COMPARISON WITH REPORTED WINDOW FUNCTION

\begin{tabular}{|c|c|c|c|c|c|c|c|c|}
\hline Existing Window Function & $\begin{array}{c}\text { Strukov } \\
{[2]}\end{array}$ & $\begin{array}{c}\text { Joglekar } \\
{[18]}\end{array}$ & $\begin{array}{c}\text { Biolek } \\
{[19]}\end{array}$ & $\begin{array}{c}\text { Prodromakis } \\
{[20]}\end{array}$ & $\begin{array}{c}\text { Shi } \\
{[24]}\end{array}$ & $\begin{array}{c}\text { Zha } \\
{[15]}\end{array}$ & $\begin{array}{c}\text { Anusudha } \\
{[16]}\end{array}$ & $\begin{array}{c}\text { Proposed } \\
\text { Function }\end{array}$ \\
\hline Resolve boundary effect & Yes & Yes & Yes & Yes & Yes & Yes & No & Yes \\
\hline Resolve boundary stick & No & No & Yes & No & Yes & Yes & Yes & Yes \\
\hline Scalability & No & No & Limited & Yes & Yes & Yes & Limited & Yes \\
\hline No. of control Parameter & Zero & One & One & Two & Two & Four & Two & Five \\
\hline Linkage with linear dopant drift model & No & Yes & Yes & Yes & Yes & Yes & Yes & Yes \\
\hline Resolve DPHL & NA & NA & No & NA & No & No & Yes & Yes \\
\hline
\end{tabular}

device very quickly, means ' $\mathrm{X}$ ' suddenly achieves their limiting value ( 0 or 1$)$. Hence, memristive device current voltage response gets distorted, that is known as distorted pinched hysteresis loop (DPHL) issue, which has been

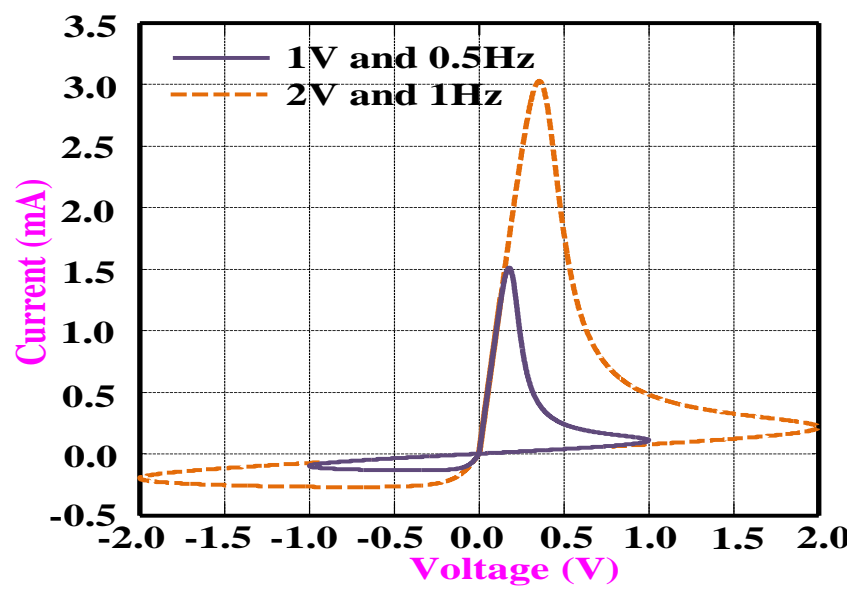

Fig. 6. Distorted I-V response of memristor for low frequency and high amplitude input voltage with $\mathrm{b}=0$.

undesirable for any application. From Fig. 6, we can see that I$\mathrm{V}$ response of the proposed model with $\mathrm{b}=0$, which is distorted in nature, for 1 Volt and $0.5 \mathrm{~Hz}$ applied stimuli and 2 Volt and

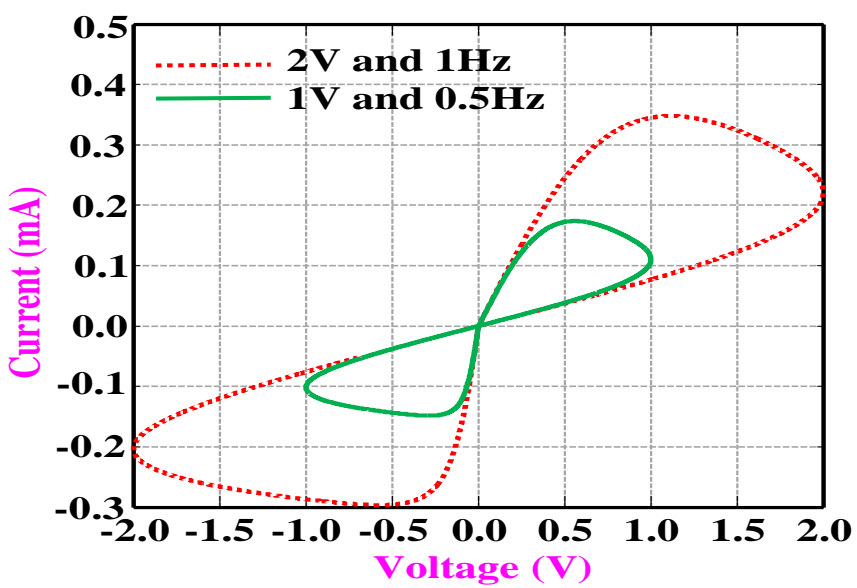

Fig. 7. Desired I-V response of memristor for low frequency and high voltage input voltage with $b=-0.6$.

$1 \mathrm{~Hz}$ applied stimuli. Further, we can evident from Fig.3, our proposed model is able to resolve DPHL issue by adjusting the window function discontinuity at its boundary with different value of control parameter ' $b$ '. In Fig. 7, we demonstrated the $\mathrm{I}-\mathrm{V}$ response of the proposed model with ' $\mathrm{b}=-0.6$ ' which is free from DPHL problem, even at low frequency or high amplitude applied voltage.

\subsection{Study of Memristance Resolution}

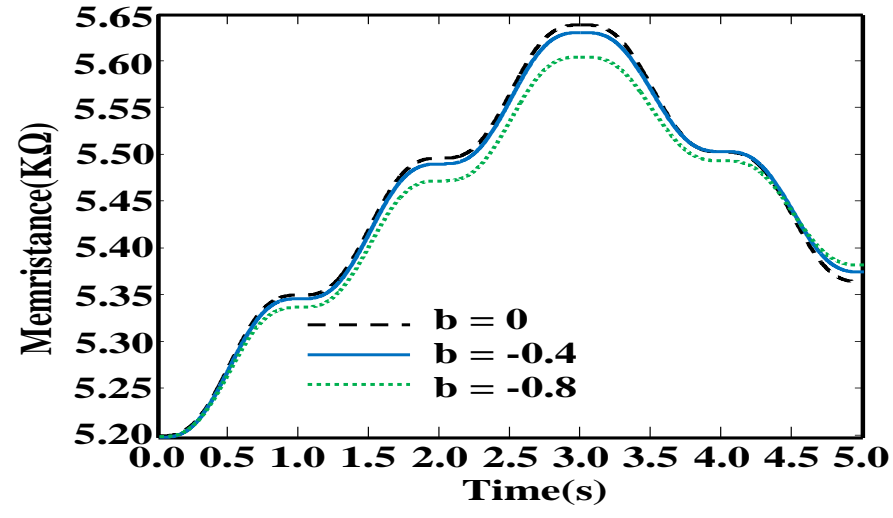

Fig. 8. Effect of ' $b$ ' on transient behavior of memristance for the unbalanced input voltage with $\mathrm{a}=0.8, \mathrm{p}=5, \mathrm{q}=1$.

The idea of memristance resolution was proposed in [25], where the value of memristance can be tuned by periodic pulses. Although, in the process of memristance tuning by periodic pulses, some certain error is exist due to the number of applied pulses should be an integer. Accordingly, designing of memristor model with high precision is very much important. As mention in [17], control parameter ' $a$ ' is able to tune the resolution of the memristance. From Fig. 8 we can see that, the additional control parameter ' $b$ ' is able to improve the memristance resolution with constant value of ' $a$ '. In our proposed model memristance tuning is analyzed by using unbalanced five sinusoidal periodic signal $v(t)=+v_{o} \sin ^{2}(2 n f * t)$ for first three pulses and $v(t)=-v_{0} \sin ^{2}(2 \pi * t)$ for next two pulses with $\mathrm{a}=0.8, \mathrm{v}_{\mathrm{o}}=0.05$ and $\mathrm{f}=1 \mathrm{~Hz}$ for different value of control parameter ' $b$ ', as demonstrated in Fig.8.

Means that, if resolution is high, then it is able to attained more number of programmable resistance state of memristor. The comparative report of proposed window model with respect to published window function model has been shown in Tabel-2. So from Table-2 we can evident that, all the issues related to the window function has been resolved by our proposed memristor window function model.

Further, in the next section we will investigate the potency of our proposed model in analog gain amplifier circuit.

\section{IMPLIMENTATION IN ANALOG GAIN AMPLIFIER CIRCUIT}

According to previous discussion, an efficient and flexile window function has been built for liner drift memristor model to resolve DPHL by adjusting discontinuity at the window function boundary and improve the resolution of memristance. Now, in this section we will illustrate the efficacy of proposed efficient window function linear drift memristor model in analog differential gain amplifier circuit.

In ordinary analog circuits, after making the circuit it is very difficult to tune the value of resistance which restricts their 
practical implementation. For resolving this issue, instead of resistor we introduce a memristor in the analog circuit which is able to tune their own resistance by applying electric pulses over it and makes it programmable [26]. In Fig.9, memristor (programmable resistor) is placed instead of resistor to control the emitter current of transistor ' $\mathrm{Q}_{3}$ ' in the analog amplifier circuit, which is responsible for tuning the gain of the analog amplifier and makes it programmable gain amplifier. This gain amplifier works in two modes: amplification and programming mode. In programming mode, resistance of the memristor is tuned to a target value by applying electrical pulse by $V_{S W}$ is set to 1 between its terminal resistance $\left(R_{\mathrm{ON}}\right.$ and $\mathrm{R}_{\mathrm{OFF}}$ ). In amplification mode, after achieving target resistance of memristor, $\mathrm{V}_{\mathrm{SW}}$ is set to 0 , then circuit goes into amplification mode and memristor works as resistor and makes a voltage divider circuit to the base of transistor ' $\mathrm{Q}_{3}$ ', that is deciding factor for gain of the amplifier by tuning emitter current of the transistor ' $\mathrm{Q}_{3}$ '. By studying small signal circuit analysis, analog amplifier differential voltage gain is formulated as

$$
\begin{aligned}
& \mathrm{A}_{\mathrm{VD}}=\mathrm{g}_{\mathrm{m}} * \mathrm{R}_{\mathrm{c}}=\left(\mathrm{I}_{\mathrm{c}} / \mathrm{V}_{\mathrm{t}}\right) * \mathrm{R}_{\mathrm{c}}=\left(\frac{\mathrm{I}_{\mathrm{e} 3}}{2 * \mathrm{~V}_{\mathrm{t}}}\right) * \mathrm{R}_{\mathrm{c}} \\
& \mathrm{I}_{\mathrm{e} 3} \approx \frac{\mathrm{V}_{\mathrm{EE}}\left(1-\frac{\mathrm{R}_{\mathrm{M}}}{\mathrm{R}_{\mathrm{M}}+\mathrm{R}}\right)-\mathrm{v}_{\mathrm{be}}}{\mathrm{R}_{\mathrm{E}}}
\end{aligned}
$$

Where $\mathrm{R}_{\mathrm{c}}=\mathrm{R}_{\mathrm{cl}}=\mathrm{R}_{\mathrm{c} 2}=3 \mathrm{~K} \Omega, \mathrm{R}_{\mathrm{E}}=2 \mathrm{~K} \Omega, \mathrm{R}=2 \mathrm{~K} \Omega$ and ' $\mathrm{I}_{\mathrm{e}}$ ' is the emitter current in the transistor ' $\mathrm{Q}_{3}$ ' which has expressed in the expression-14. In addition, memristance programming electrical pulses is same, as mention in section 4.4.The analog voltage gain circuit is simulated in SPICE where the parameter

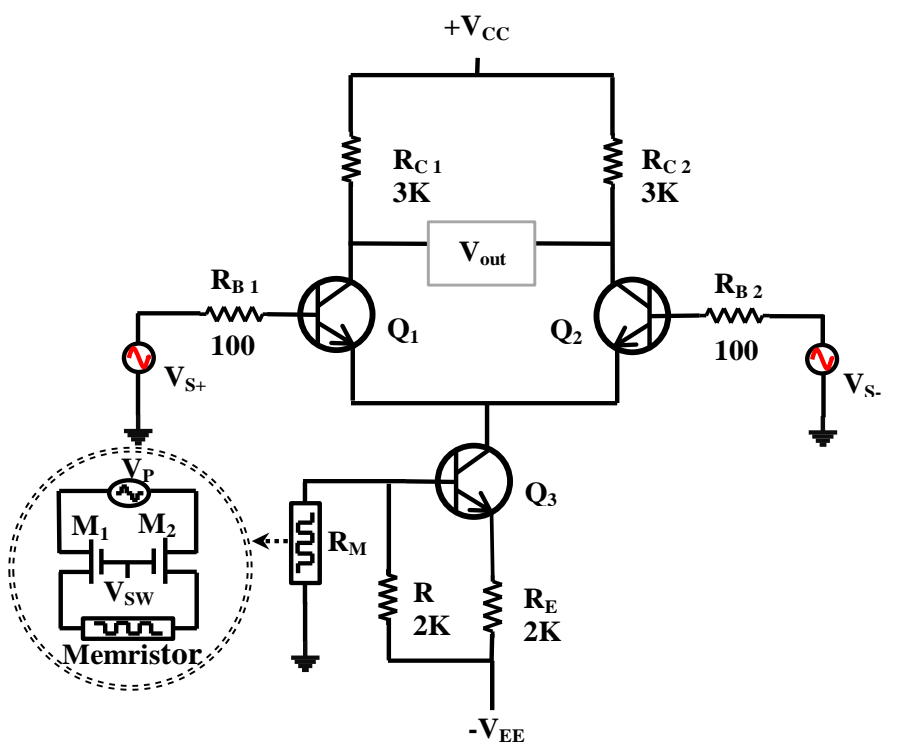

Fig. 9. A programmable analog gain amplifier

$a=0.5$ and $R_{\text {intial }}=100 \Omega$ with $b=0.0$ and $b=-0.6$. Moreover, differential mode input voltages are $\mathrm{V}_{\mathrm{s}+}=\mathrm{V}_{\mathrm{a}} \sin \left(2 \pi \mathrm{f}^{*} \mathrm{t}\right)$ and $\mathrm{V}_{\mathrm{s}-}$ $=-\mathrm{V}_{\mathrm{a}} \sin \left(2 \pi \mathrm{f}^{*} \mathrm{t}\right)$, where $\mathrm{V}_{\mathrm{a}}=20 \mathrm{mV}$ and $\mathrm{f}=1 \mathrm{KHz}$. As Table -3 shows, resistance of the memristor and the gain of the circuit closely related to the applied number of electric pulses as well as shows, for attending same memristance value, different number of electrical pulses required with different value of control parameter ' $b$ '. Since, $b=-0.6$ model required more electrical pulses then with $b=0.0$. Hence, it is clear that, $b=$ -
0.6 model can adopt more number of discrete memristace state, which reflects the programmable gain amplifier with $b=$ -0.6 control parameter value can achieves more number of programmable stage as compared to $b=0.0$ of window model. Therefore, we can conclude that, control parameter ' $b$ ' improves the resolution of the memristor, which is desirable for any programmable application.

TABLE - 3

EFFECT OF MEMRISTANCE RESOLUTION ON PROGRAMMING STAGE AMLIFIER GAIN

\begin{tabular}{|l|l|c|c|}
\hline \multicolumn{2}{|c|}{ Number of Pulses } & \multirow{2}{*}{ Memristance $(K \Omega)$} & \multirow{2}{*}{ Gain $(\mathrm{dB})$} \\
\hline For $b=0.0$ & For $b=-0.6$ & & \\
\hline initial & initial & 0.1 & 52.1 \\
\hline 836 & 954 & 4.0 & 41.6 \\
\hline 5623 & 5897 & 16.0 & 25.2 \\
\hline
\end{tabular}

\section{CONCLUSION}

This article introduced a newly flexible and efficient window function for linear drift memristor model with unique feature of controllable window function discontinuity. Proposed window function is able to reproduces some existing window functions and also resolve all the issues associated with reported window functions. By introducing a key parameter in this proposed model, functional discontinuity of the window function become controllable, by which DPHL issue has been resolved without removing device current parameter form window function and also improved the resolution of programming resistance of memristor. Ultimately, the proposed window function has been equipped in analog gain amplifier circuit and makes it programmable with enhanced number of programming gain stage of differential gain amplifier circuit; it has been validated by SPICE simulation.

\section{REFERENCES}

[1] Chua, Leon. "Memristor-the missing circuit element." IEEE Transactions on circuit theory 18, no. 5 (1971): 507-519.

[2] Strukov, Dmitri B., Gregory S. Snider, Duncan R. Stewart, and R. Stanley Williams. "The missing memristor found." nature 453, no. 7191 (2008): 80-83.

[3] Yang, J. Joshua, Matthew D. Pickett, Xuema Li, Douglas AA Ohlberg, Duncan R. Stewart, and R. Stanley Williams. "Memristive switching mechanism for metal/oxide/metal nanodevices." Nature nanotechnology 3, no. 7 (2008): 429-433.

[4] Yakopcic, Chris, Tarek M. Taha, Guru Subramanyam, Robinson E. Pino, and Stanley Rogers. "A memristor device model." IEEE electron device letters 32, no. 10 (2011): 1436-1438.

[5] Pickett, Matthew D., Dmitri B. Strukov, Julien L. Borghetti, J. Joshua Yang, Gregory S. Snider, Duncan R. Stewart, and R. Stanley Williams. "Switching dynamics in titanium dioxide memristive devices." Journal of Applied Physics 106, no. 7 (2009): 074508.

[6] Kvatinsky, Shahar, Eby G. Friedman, Avinoam Kolodny, and Uri C. Weiser. "TEAM: Threshold adaptive memristor model." IEEE transactions on circuits and systems I: regular papers 60, no. 1 (2012): 211-221.

[7] Kvatinsky, Shahar, Misbah Ramadan, Eby G. Friedman, and Avinoam Kolodny. "VTEAM: A general model for voltage-controlled memristors." IEEE Transactions on Circuits and Systems II: Express Briefs 62, no. 8 (2015): 786-790.

[8] Singh, Jeetendra, and Balwinder Raj. "Modeling of mean barrier height levying various image forces of metal-insulator-metal structure to enhance the performance of conductive filament based memristor model." IEEE Transactions on Nanotechnology 17, no. 2 (2018): 268- 
275.

[9] Rak, Adam, and György Cserey. "Macromodeling of the memristor in SPICE." IEEE Transactions on Computer-Aided Design of Integrated Circuits and Systems 29, no. 4 (2010): 632-636.

[10]Benderli, S., and T. A. Wey. "On SPICE macromodelling of TiO 2 memristors." Electronics letters 45, no. 7 (2009): 377-379.

[11]Duan, ShuKai, XiaoFang Hu, LiDan Wang, ChuanDong Li, and Pinaki Mazumder. "Memristor-based RRAM with applications." Science China Information Sciences 55, no. 6 (2012): 1446-1460..

[12]Adhikari, Shyam Prasad, Hyongsuk Kim, Ram Kaji Budhathoki, Changju Yang, and Leon O. Chua. "A circuit-based learning architecture for multilayer neural networks with memristor bridge synapses." IEEE Transactions on Circuits and Systems I: Regular Papers 62, no. 1 (2014): 215-223.

[13]Li, Junrui, Zhekang Dong, Li Luo, Shukai Duan, and Lidan Wang. "A novel versatile window function for memristor model with application in spiking neural network." Neurocomputing 405 (2020): 239-246.

[14]Wen, Shiping, Xudong Xie, Zheng Yan, Tingwen Huang, and Zhigang Zeng. "General memristor with applications in multilayer neural networks." Neural Networks 103 (2018): 142-149.

[15]Zha, Jinxiang, He Huang, and Yujie Liu. "A novel window function for memristor model with application in programming analog circuits." IEEE Transactions on Circuits and Systems II: Express Briefs 63, no. 5 (2015): 423-427.

[16]Anusudha, T. A., and S. R. S. Prabaharan. "A versatile window function for linear ion drift memristor model-A new approach." AEUInternational Journal of Electronics and Communications 90 (2018): 130139.

[17]Zha, Jinxiang, He Huang, Tingwen Huang, Jinde Cao, Ahmed Alsaedi, and Fuad E. Alsaadi. "A general memristor model and its applications in programmable analog circuits." Neurocomputing 267 (2017): 134-140.

[18] Joglekar, Yogesh N., and Stephen J. Wolf. "The elusive memristor: properties of basic electrical circuits." European Journal of physics 30, no. 4 (2009): 661.

[19] Biolek, Zdeněk, Dalibor Biolek, and Viera Biolkova. "SPICE Model of Memristor with Nonlinear Dopant Drift." Radioengineering 18, no. 2 (2009).

[20] Prodromakis, Themistoklis, Boon Pin Peh, Christos Papavassiliou, and Christofer Toumazou. "A versatile memristor model with nonlinear dopant kinetics." IEEE transactions on electron devices 58, no. 9 (2011): 3099-3105.

[21]Chen, Wanlong, Xiao Yang, and Frank Z. Wang. "An omnipotent memristor model with controllable window functions." In 2015 17th UKSim-AMSS International Conference on Modelling and Simulation (UKSim), pp. 600-605. IEEE, 2015.

[22]Mladenov, Valeri, and Stoyan Kirilov. "A nonlinear drift memristor model with a modified biolek window function and activation threshold." Electronics 6, no. 4 (2017): 77.

[23]Chowdhury, Joy, J. K. Das, and N. K. Rout. "Trigonometric window functions for memristive device modeling." In 2015 Fifth International Conference on Advanced Computing \& Communication Technologies, pp. 157-161. IEEE, 2015.

[24]Shi, Min, Yajuan Yu, and Qi Xu. "Window function for fractional-order HP non-linear memristor model." IET Circuits, Devices \& Systems 12, no. 4 (2018): 447-452.

[25]Shin, Sangho, Kyungmin Kim, and Sung-Mo Kang. "Memristor applications for programmable analog ICs." IEEE Transactions on Nanotechnology 10, no. 2 (2010): 266-274.

[26]Pershin, Yuriy V., and Massimiliano Di Ventra. "Practical approach to programmable analog circuits with memristors." IEEE Transactions on Circuits and Systems I: Regular Papers 57, no. 8 (2010): 1857-1864. 